Background and aims The PI3K pathway is frequently activated during tumourigenesis through deletion of the tumour suppressor PTEN. In contrast, increased PTEN expression in adipose tissue results in an increase in UCP1 expression and provides metabolic protection from tumourigenesis. This intrinsic protection normally arises from interscapular brown adipose tissue (iBAT) but may also arise from 'beiging' of inguinal white adipose tissue (iWAT). The aim of this study was to see if an association existed between UCP1 expression in adipose tissue and paediatric brain tumour growth through elevated PTEN levels.

Methods Two types of medulloblastoma (WNT and group 4) and ependymoma tumour cells were orthotopically xenografted into mice. iBAT and iWAT samples were extracted from tumour and non-tumour bearing mice to examine UCP1 and PTEN expression through QRT-PCR and Western blotting. Haematoxylin and eosin staining and UCP1 antibody immunohistochemistry (IHC) was also used to determine BAT activity in adipose tissue. Thermogenic activity of the adipose tissue was indirectly measured by thermal imaging of mice.

Results iWAT from ependymoma tumour-bearing mice had evidence of beiging and increased UCP1 expression through histology and IHC, while UCP1 expression in iBAT remained high in all mice. An increase in UCP1 gene expression and thermogenesis was observed with spinal metastasis. PTEN expression did not relate to UCP1 expression.

Conclusion Our data indicated mice implanted with aggressive tumours had increased UCP1 expression in iWAT. In conclusion, this pilot study suggests rapidly growing and metastatic brain tumours stimulate metabolic protection via an increase UCP1 expression in iWAT.

\section{Paediatric Emergency Medicine II}

\section{0-094 DIAGNOSTIC USEFULNESS OF BIOMARKERS IN THE MANAGEMENT OF CHILDREN WITH FEVER AT RISK OF SERIOUS BACTERIAL INFECTIONS AT THE EMERGENCY DEPARTMENT: PROSPECTIVE DIAGNOSTIC STUDY}

${ }^{1} \mathrm{RG}$ Nijman, ${ }^{2} Y$ Vergouwe, ${ }^{1} \mathrm{HA}$ Moll, ${ }^{3} \mathrm{WA}$ Dik, ${ }^{4} \mathrm{FJ}$ Smit, ${ }^{1} \mathrm{M}$ van Veen, ${ }^{5} \mathrm{~F}$ Weerkamp, ${ }^{2}$ EW Steyerberg, ${ }^{6}$ J van der Lei, ${ }^{7}$ YB de Rijke, ${ }^{1} \mathrm{R}$ Oostenbrink. ${ }^{1}$ Department of Pediatrics, Erasmus MC Sophia Children's Hospital, Rotterdam, Netherlands; ${ }^{2}$ Department of Public Health, Erasmus MC - University Medical Center Rotterdam, Rotterdam, Netherlands; ${ }^{3}$ Department of Immunology, Erasmus MC - University Medical Center Rotterdam, Rotterdam, Netherlands; ${ }^{4}$ Department of Pediatrics, Maasstad Hospital, Rotterdam, Netherlands; ${ }^{5}$ Department of Clinical Chemistry, Maasstad Hospital, Rotterdam, Netherlands; ${ }^{6}$ Department of Medical Informatics, Erasmus MC - University Medical Center Rotterdam, Rotterdam, Netherlands; ${ }^{7}$ Department of Clinical Chemistry, Erasmus MC - University Medical Center Rotterdam, Rotterdam, Netherlands

\subsection{6/archdischild-2014-307384.161}

Background and aims To evaluate the diagnostic usefulness of biomarkers in the management of children with fever at risk of serious bacterial infections (SBI) at the emergency department (ED).

Methods In this prospective observational study previously healthy children with fever, aged 1 month to 16 years, attending the EDs of a university hospital and a teaching hospital (Rotterdam, the Netherlands) between 2009 and 2012 were included. Standardised information on clinical signs and symptoms, Creactive protein (CRP), procalcitonin (PCT), neutrophil CD64 expression and urinalysis were collected prospectively. Logistic multivariable regression analysis was used to assess diagnostic performance.

Results 1,084 children were included, median age was 1.6 years (interquartile range: $0.8-3.5), 170$ children (16\%) had SBI. CRP (receiver operating characteristic curve (ROC-area) 0.77 (95\% confidence interval (CI) $0.69-0.85)$ ) and PCT (ROC-area 0.75 (95\% CI 0.67-0.83)) were both strong predictors of SBI. CD64 lacked diagnostic strength (ROC-area 0.62 (95\% CI 0.54-0.70)). A score containing PCT and CRP together with urinalysis, the Lab-score, performed well (ROC-area 0.79 (95\% CI $0.72-$ 0.87)), but thresholds performed similar to often used cut-offs of single biomarkers. Combined with clinical signs and symptoms both CRP and PCT were useful; additional PCT to CRP did not improve diagnostic performance substantially.

Conclusions CRP and PCT were equally useful in the diagnostic evaluation of the febrile child, whereas CD64 wasn't useful. Performing both CRP and PCT is often not indicated in a general population of febrile children. Our findings contrast previous studies suggesting PCT outperforming CRP and superior value of CD64 in specific settings.

\section{0-095 INFANTS WITH FRACTURES IN THE PAEDIATRIC EMERGENCY DEPARTMENT: ARE WE CONSIDERING CHILD PHYSICAL ABUSE?}

${ }^{1} \mathrm{~L}$ Lavin, ${ }^{2} \mathrm{C}$ Penrod, ${ }^{1} \mathrm{CM}$ Estrada, ${ }^{1} \mathrm{D}$ Arnold, ${ }^{3} \mathrm{X}$ Meng, ${ }^{3} \mathrm{~B}$ Saville, ${ }^{2} \mathrm{D}$ Lowen. ${ }^{1}$ Department of Pediatrics Division of Emergency Medicine, Vanderbilt University, Nashville, USA; ${ }^{2}$ Department of Pediatrics, Vanderbilt University, Nashville, USA; ${ }^{3}$ Department of Biostatistics, Vanderbilt University, Nashville, USA

\subsection{6/archdischild-2014-307384.162}

Background Recognition of child physical abuse (PA) is important to avoid further morbidity and mortality. There is limited knowledge regarding how frequently paediatric emergency department (PED) clinicians consider child abuse in infants with fractures.

Objective Estimate the percentage of infants with fractures for whom PA was considered, and to examine characteristics that are associated with consideration of PA.

Methods We reviewed the electronic medical record (EMR) of all patients $\leq 1$ year with fractures in a PED between 20082012. We used a multivariable logistic regression model to examine associations of patient and physician characteristics with our

\begin{tabular}{|c|c|}
\hline \multicolumn{2}{|l|}{ Patient characteristics } \\
\hline Age, in weeks ${ }^{a}$ & $27[12.1,38.7]$ \\
\hline Gender, Male ${ }^{\mathrm{b}}$ & $282(53)$ \\
\hline \multicolumn{2}{|l|}{ Race $^{b}$} \\
\hline Caucasian & $372(75)$ \\
\hline African American & $92(19)$ \\
\hline Hispanic & $21(4)$ \\
\hline \multicolumn{2}{|l|}{ Physician characteristics } \\
\hline Male $^{b}$ & $282(53)$ \\
\hline PEM fellowship trained ${ }^{b, c}$ & $308(58)^{c}$ \\
\hline \multicolumn{2}{|l|}{ a. Median [IQR] } \\
\hline \multicolumn{2}{|l|}{ b. $n(\%)$} \\
\hline c. All others EM-trained except 1 paediatrics-trained & \\
\hline
\end{tabular}


primary outcome variable, consideration of PA, defined as clearly-documented consideration of PA in attending or trainee note OR skeletal survey ordered OR child-abuse team consult ordered. Co-variates examined as well.

Results Characteristics of 529 patients and physicians are displayed in Table 1. For the entire cohort, consideration of $\mathrm{PA}$ occurred in 346 (65\%), whereas consideration in infants $<6$ months of age occurred in 194 (78\%). EMR clearlydocumented consideration of PA occurred in 288 (54\%). Characteristics associated with greater odds for consideration of PA after covariate adjustment (OR and 95\% CI) included younger patient age (IQR of 12.1 to 38.7 weeks: 0.53 [0.33, $0.84]$ ), no history provided for injury $(9.41[3.88,22.82])$, softtissue injury $(4.95,[1.57,15.54])$, and male attending (1.84, $[1.12,3.01])$.

Conclusion PED physicians frequently do not consider PA in infants with fractures. Characteristics associated with consideration for PA include patient age, no history provided to explain the injury, soft-tissue injury, and male attending gender.

\section{Paediatric Intensive Care}

\section{0-096 NON-RESPIRATORY PELOD-2 SCORE IS A GOOD PREDICTOR OF MORTALITY IN CHILDREN WITH ACUTE RESPIRATORY FAILURE}

${ }^{1} S$ Leteurtre, ${ }^{2} \mathrm{~A}$ Duhamel, ${ }^{2} \mathrm{~V}$ Deken, ${ }^{1} \mathrm{C}$ Le Reun, ${ }^{3} \mathrm{~J}$ Lacroix, ${ }^{4} \mathrm{~F}$ Leclerc, ${ }^{1} \mathrm{Gfrup} .{ }^{7} \mathrm{PICU}$, CHRU de Lille, LILLE Cedex, France; ' ${ }^{2}$ Department of Biostatisitics, CHRU de Lille, LILLE Cedex, France; ${ }^{3}$ PICU, Sainte Justine Hospital, Montréal, Canada; ${ }^{4}$ Paris, GFRUP: Groupe Francophone de Reanimation Et Urgences Pediatriques, France

\subsection{6/archdischild-2014-307384.163}

Background and aim Multiple organ dysfunction, not respiratory failure, is the major cause of death in children with ALI or ARDS. This study was undertaken to estimate the predictive value of death of the non-respiratory Paediatric Logistic Organ Dysfunction (PELOD)-2 (NRespPELOD-2) in children with acute respiratory failure (ARF).

Methods Analysis of the database of the recently published PELOD-2. All consecutive children (excluding neonates) admitted to 9 PICU in France and Belgium (June 2006-October 2007) and having ARF. We prospectively collected data on variables considered for the PELOD-2 score during PICU stay: days 1, 2, 5, 8, 12, 16 and 18, plus PICU discharge. For each variable of the PELOD-2 score, the most abnormal value observed during time points was collected. Outcome was vital status at PICU discharge. We used AUCs to estimate the discrimination and Hosmer-Lemeshow goodness-of-fit tests to estimate calibration of the PELOD-2 and the NRespPELOD-2 scores, with correction for the optimism bias using a bootstrap resampling method.

Results We included 1572 patients (median age: 20.6 months; mortality: 9.5\%). Discrimination of the PELOD-2 and the NRespPELOD-2 was excellent (AUC $=0.93$ and 0.92, respectively) and calibration was good ( $\mathrm{p}=0.45$ and 0.27 , respectively). The four NResp organ dysfunctions were closely related to the risk of mortality $(\mathrm{p}<0.001)$.

Conclusions Our study demonstrates that the NRespPELOD-2 score of the entire PICU stay is highly predictive of death in children with ARF of whom $94.3 \%$ were invasively ventilated. It could represent the non-respiratory organ failure definition tool claimed by the international experts on paediatric ARDS.

\section{0-097 CLINICAL SCORE TO PREDICT OUTCOME IN CONGENITAL DIAPHRAGMATIC HERNIA; RESULTS OBTAINED FROM AN INTERNATIONAL MULTICENTER STUDY}

${ }^{1} \mathrm{KG}$ Snoek, ${ }^{2} \mathrm{C}$ Capolupo, ${ }^{2} \mathrm{~F}$ Morini, ${ }^{1} \mathrm{RMH}$ Wijnen, ${ }^{3} \mathrm{~K} \mathrm{KM}$ Reiss, ${ }^{2} \mathrm{P}$ Bagolan, ${ }^{1} \mathrm{H}$ IJsselstijn, ${ }^{1} \mathrm{D}$ Tibboel. 'Department of Paediatric Surgery and Intensive Care, Erasmus MC Sophia Children's Hospital, Rotterdam, Netherlands; '2Department of Medical and Surgical Neonatology, Bambino Gesu Children's Hospital, Rome, Italy; ${ }^{3}$ Department of Neonatology, Erasmus MC Sophia Children's Hospital, Rotterdam, Netherlands

\subsection{6/archdischild-2014-307384.164}

Background In congenital diaphragmatic hernia $(\mathrm{CDH})$ reliable postnatal predictors of outcome are scarce. Score for Neonatal Acute Physiology-II (SNAP-II) (Richardson, 2001) has been validated to predict mortality in newborns. We hypothesised that higher SNAP-II scores are found in non-survivors, those who need extracorporeal membrane oxygenation (ECMO), and those who develop chronic lung disease (CLD), defined as oxygen dependency at day 28.

Methods In a prospective, randomised controlled multicenter trial initiated by the Euroconsortium (VICI-trial, NTR 1310), neonates born between November 2008 and December 2013, were randomised for initial ventilation strategy (either high-frequency oscillation or conventional mechanical ventilation). Prenatal, perinatal and postnatal data including SNAP-II scores were collected. Mann-Whitney U test was used for analyses. Data were presented as $\mathrm{n}(\%)$ and median (IQR).

Results Of the 173 included patients, 46 patients (26.6\%) died, $39(22.5 \%)$ underwent ECMO, and 38 patients (29.9\%) of the survivors developed CLD. Overall, the SNAP-II score was 22.5 (10 to 37). In non-survivors SNAPP-II score was 40.0 (32.0 to 54.5 ) and 16.5 (7.8 to 26.5 ) in survivors ( $p<0.001)$. It was 35.0 (30.0 to 46.0) in ECMO treated patients and 19.0 (9.0 to $32.0)$ in patients without ECMO need $(\mathrm{p}<0.001)$. Survivors with CLD had SNAP-II score of 25.5 (21.0 to 35.5) and those without CLD 13.0 (5.0 to21.0) $(\mathrm{p}<0.001)$.

Conclusions In this prospective multicenter study, we showed that in CDH patients SNAP-II scores may not only be useful to predict mortality but also to identify patients who need ECMO or are at risk for development of CLD.

\section{Pharmacology I}

\section{0-098 POTENTIALLY HARMFUL EXCIPIENTS IN MEDICINES PRESCRIBED IN NEONATAL INTENSIVE CARE UNITS (NICU) - PRODUCT SUBSTITUTION AS A WAY FORWARD}

${ }^{1} \mathrm{G}$ Nellis, ${ }^{2}$ Lutsar, ${ }^{1} \mathrm{H}$ Varendi, ${ }^{3} \mathrm{~J}$ Lass, ${ }^{4} \mathrm{MA}$ Turner, ${ }^{5} \mathrm{~T}$ Metsvaht. ${ }^{1}$ Neonatal Unit, Children's Clinic of Tartu University Hospital, Tartu, Estonia; ${ }^{2}$ Department of Microbiology, Tartu University, Tartu, Estonia; ${ }^{3}$ Pharmacy Department, Tartu University Hospital, Tartu, Estonia; ${ }^{4}$ Neonatal Unit, Liverpool Women's Hospital, Liverpool, UK; ${ }^{5}$ Paediatric Intensive Care Unit, Tartu University, Hospital, Tartu, Estonia

\subsection{6/archdischild-2014-307384.165}

Background Excipients are essential for many medicines. Some have been associated with significant consequences in neonates. We aimed to identify substitution possibilities among medicines used in European neonates in order to minimise the administration of potentially harmful excipients of interest (EOI).

Methods A 3-day survey recording all medicines prescribed to neonates was performed in European NICUs. Based on existing toxicity data in neonates the EOI included parabens, polysorbate 80, propylene glycol, benzoates, saccharin sodium, sorbitol, 